\title{
INTRAOPERATIVE ELECTRON RADIATION THERAPY (IOERT) IN THE MANAGEMENT OF PATIENTS WITH LOCAL RECURRENT OR ADVANCED GINECOLOGIC MALIGNANCIES: A SIX CASE REVIEW
}

F. Sousa ${ }^{1}$, L. Salgado ${ }^{1}$, B. Castro ${ }^{1}$, T. Ramos $^{1}$, C. Castro ${ }^{2}$, L. Carvalho ${ }^{1}$, O. Sousa ${ }^{1}$. 1 Portuguese Oncology Institute of Porto IPO Porto, Radiotherapy, Porto, Portugal.

${ }^{2}$ Portuguese Oncology Institute of Porto IPO Porto, Cancer Epidemiology,Porto, Portugal.

\section{Introduction}

Gynecologic malignancies are the 40 most common cancer in women with a total incidence of 9.4\%. [1] After a curative strategy around $30 \%$ will experience local cancer recurrence often with a dismal prognosis and very limited curative treatment options. [2] Some of this recurrences may be suitable for a salvation surgery. In these patients, the addition of IORT to surgical resection can increase the probability of local control. [3] IORT refers to the delivery of a single large dose of radiation to a limited volume of tissue during a surgical procedure. It is usually associated to external beam radiotherapy, before or after surgery. After gross total resection of marginally respectable lesions, IORT has the potential to sterilize remaining tumor nests. During IORT, overlying normal tissue (such as bowel or other viscera) can be manually displaced from the region at risk increasing the radiation dose with very limited or no increase of toxicity. There is scarce data on efficacy and toxicity of these combined treatment approach.

\section{Objectives}

Report our experience in locally recurrent or advanced pelvic gynecologic malignancies who received intraoperative electron beam radiotherapy (IOERT) after chemoradiation.

\section{Methods}

From April 2012 to October 2018, 6 patients with recurrent cervical cancer $(n=3)$, vaginal $(n=2)$ and endometrial $(n=1)$ were treated with IOERT. Previously unirradiated $(n=1)$ patients received preoperative chemoradiation between 45$50.4 \mathrm{~Gy}$ with cisplatin. Those who had already been irradiated $(n=5)$ received 30Gy to pelvis with concomitant cisplatin before surgery. IOERT dose ranged between 10Gy-15Gy. All data were collected retrospectively and in accordance with institutional ethical policies. The Overal Survival (OS) and the disease free progression (DSP) were calculated by the Kaplan-Meier method.

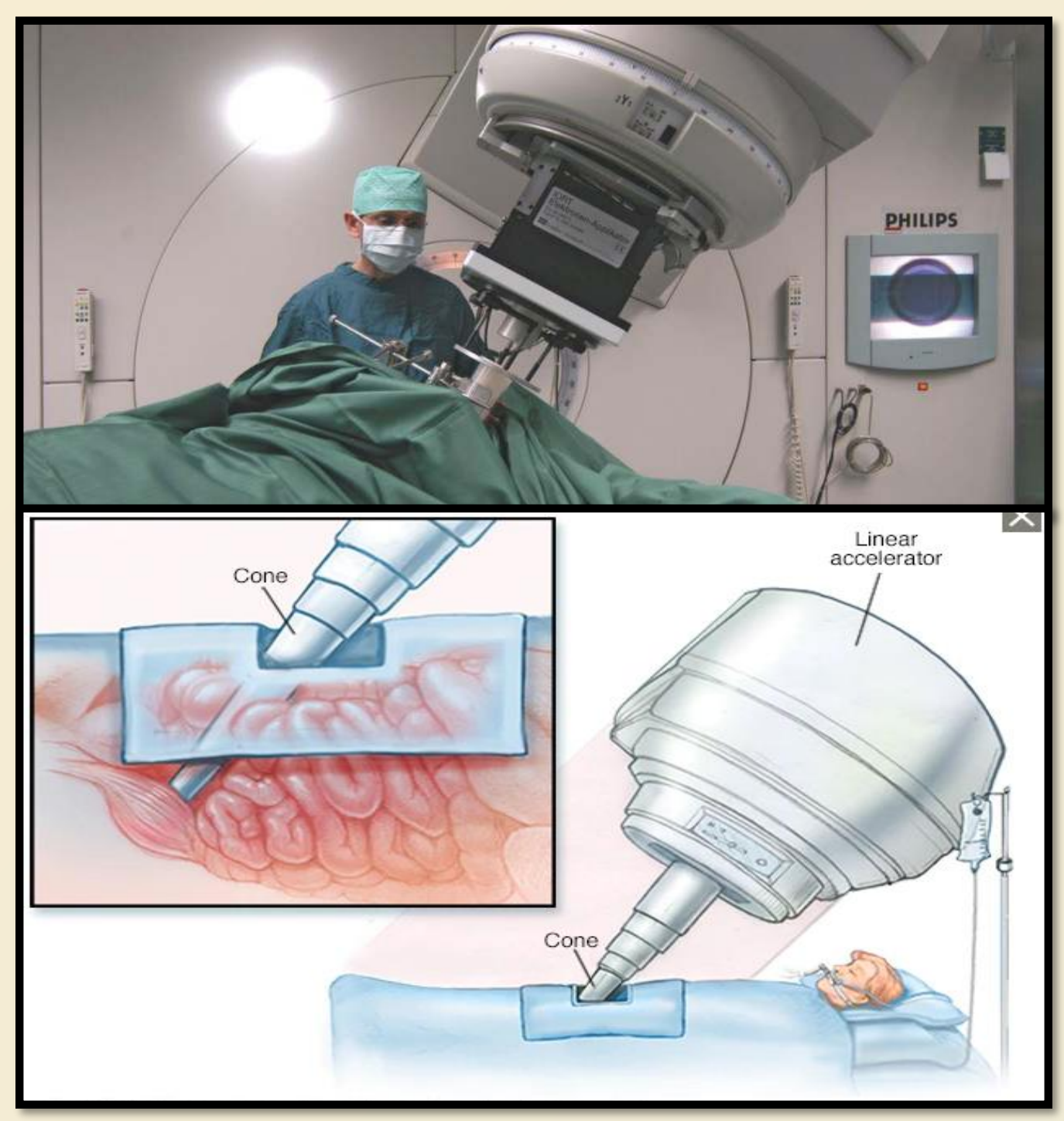

\begin{tabular}{|c|c|}
\hline & $n=6$ \\
\hline \multicolumn{2}{|l|}{ Energy } \\
\hline $6 \mathrm{MV}$ & $1(16,6 \%)$ \\
\hline $9 \mathrm{MV}$ & $5(83,3 \%)$ \\
\hline Aplicator Size Range & $6-10 \mathrm{~cm}$ \\
\hline Mean IORT duration & 17,8 min (range,11-44) \\
\hline \multicolumn{2}{|l|}{ Bisel Angle } \\
\hline $45 \underline{a}$ & $5(83,3 \%)$ \\
\hline $30 \circ$ & $1(16,6 \%)$ \\
\hline Mean hospitalization & $\begin{array}{c}23,2 \text { days ( range, } 10- \\
51 \text { ) }\end{array}$ \\
\hline $\begin{array}{l}\text { IORT related } \\
\text { toxicities }\end{array}$ & None reported \\
\hline
\end{tabular}

\section{Results}

\begin{tabular}{|c|c|c|c|c|c|c|c|c|c|c|c|c|}
\hline Case & Age & ECOG & Cancer & Histology & $\begin{array}{c}\text { Pelvic } \\
\text { Recurrence }\end{array}$ & $\begin{array}{l}\text { Time to } 10 \\
\text { recurrence } \\
\text { (months) }\end{array}$ & Surgery & EBRT & IORT & Margins & $\begin{array}{c}\text { OS } \\
\text { (months) }\end{array}$ & Recurrence \\
\hline 1 & 52 & 0 & Endometrial & $A D C$ & Central & 123,2 & Posterior Exenteration & $50,4 \mathrm{~Gy}$ & $12.5 \mathrm{~Gy}$ & RO & 43,4 & No \\
\hline 2 & 50 & 0 & Vaginal & SCQ & Lateral & 8,5 & Posterior Exenteration & 30Gy & 10Gy & RO & 85,3 & No \\
\hline 3 & 44 & 0 & Cervical & $A D C$ & Central & 38,4 & Total Exenteration & 30Gy & $12.5 \mathrm{~Gy}$ & RO & 33,3 & No \\
\hline 4 & 51 & 0 & Vaginal & SCQ & Lateral & 6,7 & Total Exenteration & $30 G y$ & $12.5 \mathrm{~Gy}$ & RO & 59,8 & No \\
\hline 5 & 56 & 1 & Cervical & $A D C$ & Lateral & 24,2 & Posterior Exenteration & 30Gy & $15 G y$ & R1 & 6,7 & No \\
\hline 6 & 50 & 0 & Cervical & $A D C$ & Lateral & 17,4 & Total Exenteration & 30Gy & $12.5 \mathrm{~Gy}$ & RO & 38,4 & No \\
\hline
\end{tabular}

ADC: Adenocarcinoma; SCQ: Squamous

\section{Conclusion}

Radical resection combined with IOERT seems to be a valid curative treatment option for patients who have failed prior surgery and/or definitive radiation. The patient selection is crucial and in addition to consideration of disease related morbidity, other factors shall be considered including the time interval from initial therapy to recurrence and whether the patient is able to receive perioperative chemoradiation and pelvic exenteration in addition to IOERT.

\begin{tabular}{|lc|}
\hline Follow-up & \\
\hline Mean follow-up & 55 months \\
\hline 3-year Overal Survival & $100 \%$ \\
\hline 3-year Disease Free Progression & $100 \%$ \\
\hline Mean time between diagnosis and recurrence & 3.6 years \\
\hline
\end{tabular}

